\title{
Light Curve Models for SN 2009dc
}

\author{
Yasuomi Kamiya \\ Department of Astronomy, Graduate School of Science, the University of Tokyo, \\ 7-3-1 Hongo, Bunkyo-ku, Tokyo 113-0033, Japan \\ Institute for the Physics and Mathematics of the Universe, \\ Todai Institutes for Advanced Study, the University of Tokyo, \\ 5-1-5 Kashiwanoha, Kashiwa, Chiba 277-8583, Japan \\ email: yasuomi.kamiya@ipmu.jp
}

\begin{abstract}
Simplified explosion models of super-Chandrasekhar-mass C-O white dwarfs (WDs) are constructed with parameters such as WD mass and ${ }^{56} \mathrm{Ni}$ mass. Their light curves are obtained by solving one-dimensional equations of radiation hydrodynamics, and compared with the observations of SN 2009dc, one of the overluminous Type Ia supernovae, to estimate its properties. As a result, the progenitor of SN $2009 \mathrm{dc}$ is suggested to be a $2.2-2.4-M_{\odot} \mathrm{C}-\mathrm{O} \mathrm{WD}$ with $1.2-1.4 M_{\odot}$ of ${ }^{56} \mathrm{Ni}$, if the extinction by its host galaxy is negligible.
\end{abstract}

Keywords. supernovae: individual (SN 2009dc) — radiative transfer — white dwarfs

SN 2009dc is one of the overluminous Type Ia supernovae (SNe Ia) and is estimated to have $\geqslant 1.2 M_{\odot}$ of ${ }^{56} \mathrm{Ni}$ (e.g., Yamanaka et al. 2009). To explain the production of such a large mass of ${ }^{56} \mathrm{Ni}$, its progenitor is proposed to be a super-Chandrasekhar-mass (super-Ch-mass) C-O white dwarf (WD). An asymmetric explosion of a Ch-mass C-O WD could also explain the high luminosity of SN 2009dc (Hillebrandt et al. 2007), but is unlikely because of the observed small polarization for SN 2009dc (Tanaka et al. 2010).

To study the properties of SN 2009dc from its light curves (LCs), simplified explosion models of super-Ch-mass C-O WDs are constructed in a manner similar to Maeda \& Iwamoto (2009). A model is parameterized by its masses of WD, electron-captured (stable Fe-peak) elements (ECEs; Fe, Co, and ${ }^{58} \mathrm{Ni}$ ), ${ }^{56} \mathrm{Ni}$, intermediate-mass elements (IMEs; $\mathrm{Si}, \mathrm{S}, \mathrm{Ca})$, and $\mathrm{C}$ and $\mathrm{O}\left(M_{\mathrm{WD}}, M_{\mathrm{ECE}}, M_{\mathrm{Ni}}, M_{\mathrm{IME}}\right.$, and $M_{\mathrm{CO}}$, respectably). Once the above parameters are set, its kinetic energy $\left(E_{\text {kin }}\right)$ is calculated, assuming that the central density is $3 \times 10^{9} \mathrm{~g} \mathrm{~cm}^{-3}$. Then the density and velocity structures are obtained by scaling those of W7 (Nomoto et al. 1984, Thielemann et al. 1986) with $M_{\mathrm{WD}}$ and $E_{\mathrm{kin}}$. For its abundance distribution, the model consists of ECEs, ${ }^{56} \mathrm{Ni}$, IMEs, and C-O layers, from the center to the surface. However, mixing is assumed for a certain region due to the observed slow Si II line velocity (Yamanaka et al. 2009). The parameter ranges in this study are $M_{\mathrm{WD}} / M_{\odot}=1.8,2.0, \ldots, 2.8 ; M_{\mathrm{Ni}} / M_{\odot}=1.2,1.4,1.6,1.8 ; M_{\mathrm{ECE}} / M_{\mathrm{WD}}=0.1,0.2, \ldots ;$ and $M_{\mathrm{CO}} / M_{\mathrm{WD}}=0.1,0.2, \ldots$ With the relation, $M_{\mathrm{WD}}=M_{\mathrm{ECE}}+M_{\mathrm{Ni}}+M_{\mathrm{IME}}+M_{\mathrm{CO}}$, $M_{\mathrm{IME}}$ is hereafter not indicated. Any model whose $E_{\mathrm{kin}}$ is not positive is excluded.

The STELLA code is used to calculate the LCs, which solves one-dimensional equations of radiation hydrodynamics (e.g., Blinnikov et al. 1998). In this study, multi-band ( $U-, B-$, $V$-, $R$-, and $I$-band) LCs are obtained, as well as bolometric ones. The "bolometric" LCs in the observations are not truly bolometric, but cover uvoir. For SN 2009dc, the observed uvoir luminosity is assumed to be $\sim 1.6$ the integrated BVRI one (Yamanaka et al. 2009), which is common for normal SNe Ia (Wang et al. 2009). Since it is still unknown whether this assumption is true for overluminous SNe Ia, BVRI LCs are compared in the following.

The resultant $B V R I$ LCs of the models show a similar tendency to that in Maeda \& Iwamoto (2009); a massive WD model has a wider BVRI LC and vice versa. Since SN 

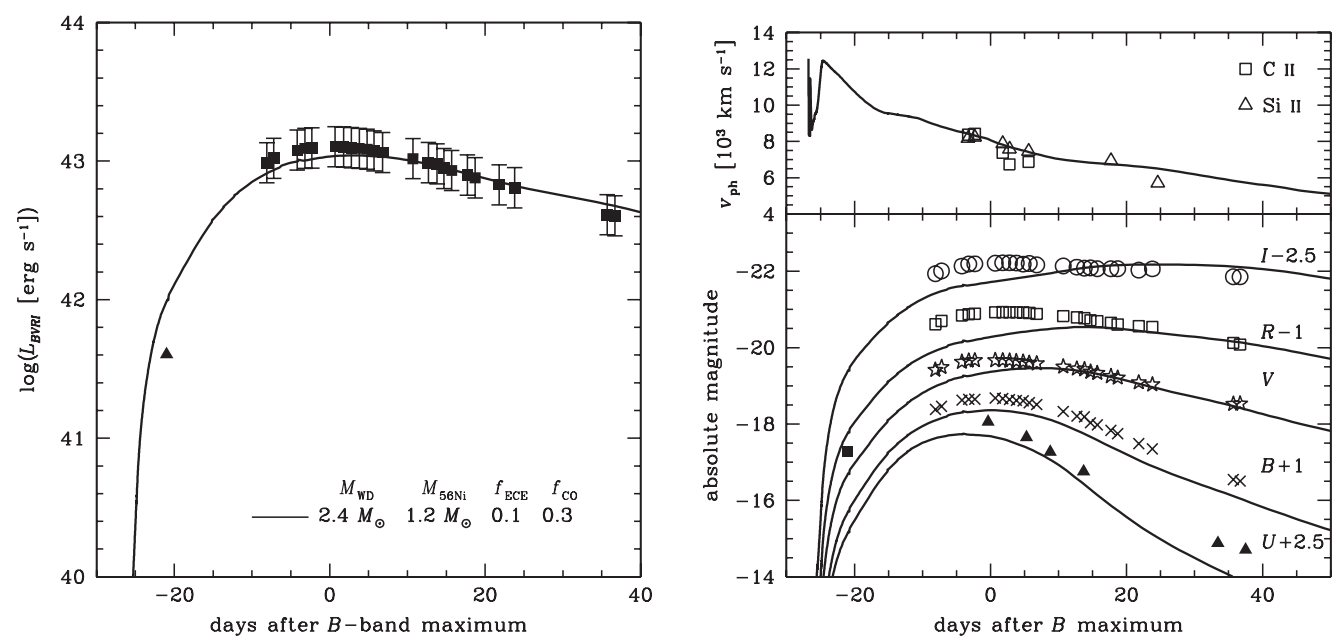

Figure 1. Comparison of the most preferred model with SN 2009dc. The model parameters are indicated in the left panel. The observational data are taken from Yamanaka et al. (2009), except for the early detection (triangle in the left panel, filled square in the right-bottom), estimated from Silverman et al. (2011). Note that the extinction by the host galaxy is neglected.

2009dc shows a wide $B V R I$ LC, lighter WD models such as $M_{\mathrm{WD}}=1.8 M_{\odot}$ can be excluded. Many models have much larger photospheric velocity $\left(v_{\mathrm{ph}}\right)$ than the observed line velocity. To make a further comparison between the BVRI LCs of the models and the observations without the host-galaxy extinction, $\chi^{2}$ is calculated for the models with smaller $v_{\mathrm{ph}}$. Among them, the models with $M_{\mathrm{WD}}=2.2-2.4 M_{\odot}, M_{\mathrm{Ni}}=1.2-1.4 M_{\odot}$, $M_{\mathrm{ECE}}=0.1 M_{\mathrm{WD}}$, and $M_{\mathrm{CO}}=0.3 M_{\mathrm{WD}}$ show relatively small $\chi^{2}$, being well fitted to the observation (Figure 1). These models indicate that the progenitor of SN 2009dc should have a relatively massive $\mathrm{C}-\mathrm{O}$ layer, which results in smaller $E_{\mathrm{kin}}$ and thus $v_{\mathrm{ph}}$.

\section{Acknowledgements}

Y.K. is grateful to his collaborators, Masaomi Tanaka, Ken'ichi Nomoto, Sergei I. Blinnikov, Elena I. Sorokina, and Tomoharu Suzuki. As a research fellow of the Japan Society for the Promotion of Science (JSPS), he and his work are financially supported by the JSPS Research Fellowships for Young Scientists and Grant-in-Aid for JSPS Fellows \#22.7641, respectably.

\section{References}

Blinnikov, S. I., Eastman, R., Bartunov, O. S., et al. 1998, ApJ, 496, 454

Hillebrandt, W., Sim, S. A., \& Röpke, F. K. 2007, A\&A (Letters), 465, L17

Maeda, K. \& Iwamoto, K. 2009, MNRAS, 394, 239

Nomoto, K., Thielemann, F.-K., \& Yokoi, K. 1984, ApJ, 286, 644

Silverman, J. M., Ganeshalingam, M., Li, W., et al. 2011, MNRAS, 410, 585

Tanaka M., Kawabata K. S., Yamanaka M., et al. 2010, ApJ, 714, 1209

Thielemann, F.-K., Nomoto, K., \& Yokoi, K. 1986, A\&A, 158, 17

Wang, X., Li, W., Filippenko, A. V., et al. 2009, ApJ, 697, 380

Yamanaka M., Kawabata K. S., Kinugasa K., et al. 2009, ApJ (Letters), 707, L118 